University of Texas at El Paso

ScholarWorks@UTEP

2-2019

\title{
Derivation of Louisville-Bratu-Gelfand Equation from Shift- or Scale-Invariance
}

Leobardo Valera

The University of Texas at El Paso, leobardovalera@gmail.com

Martine Ceberio

The University of Texas at El Paso, mceberio@utep.edu

Vladik Kreinovich

The University of Texas at El Paso, vladik@utep.edu

Follow this and additional works at: https://scholarworks.utep.edu/cs_techrep

Part of the Computer Sciences Commons

Comments:

Technical Report: UTEP-CS-19-08

\section{Recommended Citation}

Valera, Leobardo; Ceberio, Martine; and Kreinovich, Vladik, "Derivation of Louisville-Bratu-Gelfand Equation from Shift- or Scale-Invariance" (2019). Departmental Technical Reports (CS). 1286.

https://scholarworks.utep.edu/cs_techrep/1286

This Article is brought to you for free and open access by the Computer Science at ScholarWorks@UTEP. It has been accepted for inclusion in Departmental Technical Reports (CS) by an authorized administrator of ScholarWorks@UTEP.For more information, please contact Iweber@utep.edu. 


\title{
Derivation of Louisville-Bratu-Gelfand Equation from Shift- or Scale-Invariance
}

Leobardo Valera, Martine Ceberio, and Vladik Kreinovich

\begin{abstract}
Louisville-Bratu-Gelfand equation appear in many different physical situations ranging from combustion to explosions to astrophysics. The fact that the same equation appears in many different situations seems to indicate that this equation should not depend on any specific physical process, that it should be possible to derive it from general principles. This is indeed what we show in this paper: that this equation can be naturally derived from basic symmetry requirements.
\end{abstract}

\section{Formulation of the Problem}

In many different situations, we have the exact same Louisville-Bratu-Gelfand equation. In many different physical situations, we encounter the same differential equation

$$
\nabla^{2} \varphi=c \cdot \exp (a \cdot \varphi) .
$$

This equation - known as Louisville-Bratu-Gelfand equation - appears in the analysis of explosions, in the study of combustion, in astrophysics (to describe the matter distribution in a nebula), in electrodynamics - to describe the electric space charge around a glowing wire - and in many other applications areas; see, e.g., $[2,3,5,6,7,8,10,11]$.

Challenge. The fact that the same equation appears in many different situations seems to indicate that this equation should not depend on any specific physical process, that it should be possible to derive it from general principles.

What we do in this paper. In this paper, we show that this equation can be naturally derived from basic symmetry requirements.

Leobardo Valera, Martine Ceberio, and Vladik Kreinovich

Department of Computer Science, University of Texas at El Paso, El Paso, Texas 79968, USA

e-mail: leobardovalera@gmail.com,mceberio@utep.edu,vladik@utep.edu 


\section{Laplace Equation - the Simplest Case of Louisville-Bratu-Gelfand Equation: Brief Reminder}

Idea. The simplest form of equation (1) is when we take $c=0$. In this case, we get a linear equation $\nabla^{2} \varphi=0$. This equation is known as the Laplace equation. So, in order to understand where the equation (1) comes from, let us first recall where the Laplace equation comes from.

Scalar fields are ubiquitous. To describe the state of the world, we need to describe the values of all the physical quantities at different locations. In physics, the dependence $\varphi(x)$ of a physical quantity $\varphi$ on the location $x$ is known as a field. Typical examples are components of an electric or magnetic fields, gravity field, etc.

In general, at each location $x$, there are many different physical fields. In some cases, we need to take all of them - or at least several of them - into account, since several fields are strong enough to affect the situation. However, in many practical situations, only one field is strong enough.

For example, when we analyze the motion of celestial bodies, we can safely ignore all the fields except for gravity. Similarly, if we analyze electric circuits, we can safely ignore all the fields but the electromagnetic field.

Case of weak fields. In general, equations describing fields are non-linear. However, in many real-life situations, fields are weak. In this case, we can safely ignore quadratic and higher order terms in terms of $\varphi$ and consider linear equations.

General case of linear equations. In physics, usually, we consider second order differential equations; see, e.g., [4, 9], i.e., equations that depends on the field $\varphi$, on its first order partial derivatives $\varphi_{, i} \stackrel{\text { def }}{=} \frac{\partial \varphi}{\partial x_{i}}$ and on its second order derivatives $\varphi_{, i j} \stackrel{\text { def }}{=} \frac{\partial^{2} \varphi}{\partial x_{i} \partial x_{j}}$. The general linear equation containing these terms has the form

$$
\sum_{i=1}^{3} \sum_{j=1}^{3} a_{i j} \cdot \varphi_{, i j}+\sum_{i=1}^{3} a_{i} \cdot \varphi_{, i}+a \cdot \varphi=0
$$

Rotation-invariance. In general, physics does not change if we simply rotate the coordinate system. Thus, it is reasonable to require that the system (2) be invariant with respect to arbitrary rotations. This requirement eliminates the terms proportional to the first derivatives $\varphi_{, i}$ - since otherwise, we have a selected vector $a_{i}$ and thus, an expression which is not rotation-invariant.

Similarly, we cannot have different eigenvector of the matrix $a_{i j}$ - this would violate rotation-invariance. Thus, this matrix must be proportional to the unit matrix with components $\delta_{i j}$ which are equal to 1 when $i=j$ and to 0 when $i \neq j$. So, $a_{i j}=a_{0} \cdot \delta_{i j}$ for some $a_{0}$, and the equation (2) takes the form 


$$
a_{0} \cdot \sum_{i=1}^{3} \varphi_{, i i}+a \cdot \varphi=0
$$

Dividing both sides by $a_{0}$ and taking into account that $\sum_{i=1}^{3} \varphi_{, i i}=\nabla^{2} \varphi$, we get the equation of the type

$$
\nabla^{2} \varphi+m \cdot \varphi=0
$$

where we denoted $m \stackrel{\text { def }}{=} a / a_{0}$.

The equation (4) is indeed the general physics equation for a weak scalar field. The case of $m=0$ corresponds to electromagnetic field or gravitational field - or, more generally, to any field whose quanta have zero rest mass, like photons or gravitons, quanta of the above fields. In the general case, when the quanta have non-zero rest mass, we get a more general equation (4) with $m \neq 0-$ e.g., for strong interactions whose quanta are $\pi$-mesons [4, 9].

Additional conditions are needed to pinpoint Laplace equation. To explain why, out of all possible equations of type (4), Laplace equation - corresponding to $m=0$ - is the most frequent, we need to use additional conditions. As such conditions, we will use the fundamental notions of scale- and shift-invariance.

Scale-invariance: general idea. Equations deal with numbers. To describe the value of a physical quantity as a number, we need to select a measuring unit. If we change the original unit to a one which is $\lambda$ times small, then the same physical quantity which was previously described by the number $x$ will now be described by a $\lambda$ times larger number $x^{\prime}=\lambda \cdot x$. For example, if we replace meters with a 100 times smaller unit - centimeter - all the length values are multiplied by 100: 1.7 meters becomes $1.7 \cdot 100=170$ centimeters.

The choice of a measuring unit is a rather arbitrary procedure. It is therefore reasonable to require that the fundamental physical equations should not change if we simply change a measuring unit - i.e., if we replace all numerical values $x$ of the corresponding quantity to re-scaled values $x^{\prime}=\lambda \cdot x$.

Of course, different quantities may be related, so if we change the unit of one quantity, we may need to appropriate change units for measuring related quantities. For example, if we change the unit of time $t$, e.g., for hours to seconds, then, to preserve the relation $d=v \cdot t$ between the velocity $v$ and the distance $d$, we need to also change the unit for measuring velocity - e.g., from kilometers per hour to kilometers per second.

Two quantities. Equation (4) involves two physical quantities: the physical field $\varphi$ and the coordinate (distance) $x_{i}$. Thus, we can consider scale-invariance with respect to both these quantities.

$\varphi$-scale-invariance. Since the equation (4) is linear in $\varphi$, it clearly does not change if we replace the original field $\varphi(x)$ with a $\varphi$-re-scaled field $\varphi^{\prime}(x)=\lambda \cdot \varphi(x)$.

$x$-scale-invariance. If we change the unit of measuring $x_{i}$ to a unit which is $\lambda$ times smaller, then the numerical values will change from $x_{i}$ to $\lambda \cdot x_{i}$. Thus, each derivative 
$\frac{\partial}{\partial x_{i}}$ gets divided by $\lambda$, and so, the second derivative is divided by $\lambda^{2}$, while the term $m \cdot \varphi$ remains unchanged. As a result, the equation (4) changes into

$$
\frac{1}{\lambda^{2}} \cdot \nabla^{2} \varphi+m \cdot \varphi=0
$$

or, equivalently, into

$$
\nabla^{2} \varphi+m \cdot \lambda^{2} \cdot \varphi=0
$$

The only case when this equation is equivalent to the original equation (4) is when the coefficients at $\varphi$ in the equations (4) and (5) are the same, i.e., when $m=m \cdot \lambda^{2}$ and thus, $m=0$.

It should be noticed that since re-scaling $\varphi$ does not change the equation (4), if $m \neq 0$, the equations remains different no matter how we re-scale $\varphi$.

In other words, the only $x$-scale-invariant case of the general linear equation (4) is the Laplace equation.

Shift-invariance: general idea. For many physical quantities such as time or coordinate, the numerical value also depends on the selection of the starting point. If we change the starting point of measuring time to a new one which is $s$ moments before, then, instead of the original measurement results $t$, we will new shifted numerical values $t^{\prime}=t+s$.

The selection of a starting point is simply a matter of convenience, there is nothing fundamental about it. It is therefore reasonable to require that the fundamental physical equations do not change if we simply change the starting point. Of course, to preserve the equations, we may need to accordingly change something (measuring unit or a starting point) for some other quantities.

Let us see what we can conclude in our case by requiring shift-invariance for $x_{i}$ and for $\varphi$.

$x$-shift-invariance. If we replace the original variables $x_{i}$ with new variables $x_{i}^{\prime}=$ $x_{i}+s_{i}$, where $s_{i}$ denote the corresponding shifts, then the derivatives do not change and thus, the equation (4) remains the same.

$\varphi$-shift-invariance. Let us now consider the consequences of requiring that the equation are invariant with respect to shifting the field $\varphi$, i.e., with respect to replacing all the values $\varphi(x)$ with the new values $\varphi^{\prime}(x)=\varphi(x)+s$.

In many cases, such a shift makes perfect physical sense: indeed, e.g., the only way we measure electric potential $\varphi(x)$ is by measuring the difference $\varphi(x)-\varphi\left(x^{\prime}\right)$ between potentials at different locations. If we add the same value $s$ to all the values of the field, then the differences remain the same - and thus, this addition will not affect any measurement results.

What happens if we apply this shift to the equation (4)? The derivatives do not change (since the derivative of a constant $s$ is 0 ), but the term $m \cdot \varphi$ changes into $m \cdot(\varphi+s)$. Thus, instead of the original equation (4), we get a new equation

$$
\nabla^{2} \varphi+m \cdot \varphi+m \cdot s=0 .
$$


The only possibility for the resulting equation (6) to be equivalent to (4) is when the additional term $m \cdot s$ is equal to 0 , i.e., when $m=0$.

In other words, the only $\varphi$-shift-invariant case of the general linear equation (4) is the Laplace equation.

Summary. To get Laplace equation $\nabla^{2} \varphi=0$ out of the general linear equation (4), we need to postulate either $x$-scale-invariance or $\varphi$-shift-invariance.

\section{Derivation of Louisville-Bratu-Gelfand Equation}

From Laplace equation to Poisson equation. The Laplace equation $\nabla^{2} \varphi=0$ describes what happens in the absence of any external sources. If there is an external source for the field $\varphi$, then the expression $\nabla^{2} \varphi$ is, in general, not necessarily equal to 0 . In other words, we have an equation of the type $\nabla^{2} \varphi=f$ for some external function $f$. This equation is known as the Poisson equation.

How to describe non-linearity. Non-linearity also means that the original linear equation is no longer exactly true, there are additional nonlinear terms in this equation. We can view these non-linear terms as a source for the field, i.e., in effect, we have the Poisson equation - with the only difference that now, the source term $f$ is not an external term, it is a nonlinear function of the field itself:

$$
\nabla^{2} \varphi=f(\varphi)
$$

The question is: which function $f(\varphi)$ should we choose?

Which function $f(\varphi)$ should we choose? Let us use symmetries. A natural idea for selecting the function $f(\varphi)$ is to use the same natural symmetries that we used to derive the Laplace equation in the first place: $\varphi$-shift-invariance and $x$-scaleinvariance.

When is the resulting equation $\varphi$-shift-invariant? If we replace the original values of the field $\varphi(x)$ with the shifted values $\varphi^{\prime}(x)=\varphi(x)+s$, then the derivatives will not change, so the equation (7) will take the form

$$
\nabla^{2} \varphi=f(\varphi+s)
$$

Literally speaking, these two equations coincide if $f(\varphi+s)=f(\varphi)$ for all $\varphi$ and $s$, in which case, as we can easily see, the function $f$ is simply a constant - so there is no nonlinearity.

However, as we have mentioned earlier, invariance does not mean that the equation remains the same without changing any other numerical values: sometimes, to preserve the equation, we need to accordingly make changes with other variables as well. In our case, this means that, to preserve the equation (7), in addition to a shift $\varphi \rightarrow \varphi+s$, we may also need to apply an appropriate re-scaling of the coordinates $x_{i}: x_{i} \rightarrow \lambda(s) \cdot x_{i}$. Under this re-scaling, the second derivatives are divided by $\lambda^{2}$, so, 
instead of the equation (8), we get a more complicated equation

$$
\frac{1}{\lambda^{2}(s)} \cdot \nabla^{2} \varphi=f(\varphi+s),
$$

or, equivalently,

$$
\nabla^{2} \varphi=\lambda^{2}(s) \cdot f(\varphi+s) .
$$

For the new equation (9) to be equivalent to the original equation (7), we need to make sure that their right-hand sides coincide, i.e., that for all $\varphi$ and $s$, we have

$$
\lambda^{2}(s) \cdot f(\varphi+s)=f(\varphi),
$$

or, equivalently,

$$
f(\varphi+s)=C(s) \cdot f(\varphi)
$$

where we denoted $C(s) \stackrel{\text { def }}{=} \frac{1}{\lambda^{2}(s)}$.

In physics, all dependencies are measurable, so the function $f(\varphi)$ is measurable. Thus, the function $C(s)=f(\varphi+s) / f(\varphi)$ is also measurable, as the ratio of two measurable functions. It is known (see, e.g., [1]) that for measurable functions, the only solutions to the functional equation (10) are functions

$$
f(\varphi)=c \cdot \exp (a \cdot \varphi) .
$$

This is exactly Louisville-Bratu-Gelfand equation that we are trying to explain! Thus, we arrive at the following conclusion.

Preliminary conclusion. The Louisville-Bratu-Gelfand equation can be uniquely determined if we require $\varphi$-shift-invariance.

What if we require $x$-scale-invariance? What if now, instead of the $\varphi$-shiftinvariance, we require $x$-scale-invariance?

If we replace the original values of the coordinates $x_{i}$ with the re-scaled values $x_{i}^{\prime}=\lambda \cdot x_{i}$, then the derivatives will divide by $\lambda^{2}$, while the term $f(\varphi)$ will not change. So the equation (7) will take the form

$$
\frac{1}{\lambda^{2}} \cdot \nabla^{2} \varphi=f(\varphi)
$$

i.e., equivalently,

$$
\nabla^{2} \varphi=\lambda^{2} \cdot f(\varphi)
$$

Literally speaking, these two equations coincide if $f(\varphi)=\lambda^{2} \cdot f(\varphi)$ for all $\varphi$ and $\lambda$, in which case, as we can easily see, the function $f$ is simply $0-$ so there is no nonlinearity.

However, as we have mentioned earlier, invariance does not mean that the equation remains the same without changing any other numerical values: sometimes, to preserve the equation, we need to accordingly make changes with other variables as well. In our case, this means that, to preserve the equation (7), in addition to a 
re-scaling $x_{i} \rightarrow \lambda \cdot x_{i}$, we may also need to apply an appropriate shift $s(\lambda)$ of the $\varphi$-field: $\varphi(x) \rightarrow \varphi(x)+s(\lambda)$. Under this shift, the derivatives do not change, but the value $f(\varphi)$ is replaced by the value $f(\varphi+s(\lambda))$. So, instead of the equation (11), we get a more complicated equation

$$
\frac{1}{\lambda^{2}} \cdot \nabla^{2} \varphi=f(\varphi+s(\lambda))
$$

or, equivalently,

$$
\nabla^{2} \varphi=\lambda^{2} \cdot f(\varphi+s(\lambda)) .
$$

For the new equation (12) to be equivalent to the original equation (7), we need to make sure that their right-hand sides coincide, i.e., that for all $\varphi$ and $\lambda$, we have

$$
\lambda^{2} \cdot f(\varphi+s(\lambda))=f(\varphi),
$$

or, equivalently,

$$
f(\varphi+s(\lambda))=\lambda^{-2} \cdot f(\varphi) .
$$

For this equation, we also get $f(\varphi)=c \cdot \exp (a \cdot \varphi)$ [1], i.e., we also get the Louisville-Bratu-Gelfand equation.

General conclusion. The simplest case of the Louisville-Bratu-Gelfand equation is the Laplace equation $\nabla^{2} \varphi=0$. To derive this equation from the general linear equation, we need to require either $\varphi$-shift-invariance or $x$-scale-invariance.

It turns out that in the nonlinear case, each of these two invariance requirements uniquely determines the Louisville-Bratu-Gelfand equation. The fact that this equation can be derived from natural symmetries explains why this same equation emerges in the description of many different physical phenomena.

Acknowledgements This work was supported in part by the US National Science Foundation grant HRD-1242122 (Cyber-ShARE Center of Excellence).

\section{References}

1. J. Aczél and J. Dhombres, Functional Equations in Several Variables, Cambridge University Press, 2008.

2. H. Bateman, Partial Differential Equations of Mathematical Physics, Cambridge University Press, Cambridge, UK, 1932.

3. G. Bratu, "Sur les équations intégrales non linéaires", Bulletin de la Société Mathématique de France, 1914, Vol. 42, pp. 113-142.

4. R. Feynman, R. Leighton, and M. Sands, The Feynman Lectures on Physics, Addison Wesley, Boston, Massachusetts, 2005.

5. I. M. Gelfand, "Some problems in the theory of quailinear equations", American Mathematical Society Translations, 1963, Vol. 29, No. 2, pp. 295-381.

6. D. D. Joseph and T. S. Lundgren, "Quasilinear Dirichlet problems driven by positive sources", Archive for Rational Mechanics and Analysis, 1973, Vol. 49, No. 4, pp. 241-269. 
7. J. Liouville, "Sue l'équation aux différences partielles $\frac{d^{2} \log \lambda}{d u d v} \pm \frac{\lambda}{2 a^{2}}=0$ ", Journal de Mathématiques Pures et Appliquées, 1853, pp. 71-72.

8. O. W. Richardson, The Emission of Electricity from Hot Bodies, Longmans, Green, and Co., London, 1921.

9. K. S. Thorne and R. D. Blandford, Modern Classical Physics: Optics, Fluids, Plasmas, Elasticity, Relativity, and Statistical Physics, Princeton University Press, Princeton, New Jersey, 2017.

10. L. Valera and M. Ceberio, "Model-order reduction using interval constraint solving techniques", Journal of Uncertain Systems, 2017, Vol. 11, No. 2, pp. 84-103.

11. G. W. Walker, "Some problems illustrating the forms of nebulae", Proceedings of the Royal Society of London, Series A, 1915, Vol. 91, No. 631, pp. 410-420. 